\title{
BIOCOMBUSTÍVEIS E A ECONOMIA VERDE INCLUSIVA: BUSCANDO O DESENVOLVIMENTO REGIONAL SUSTENTÁVEL NA AMAZÔNIA LEGAL BRASILEIRA ${ }^{1}$
}

\author{
BIOFUELS AND THE INCLUSIVE GREEN ECONOMY: \\ SEARCHING FOR SUSTAINABLE REGIONAL DEVELOPMENT \\ IN THE BRAZILIAN LEGAL AMAZON
}

\author{
Marcus Vinicius Alves Finco \\ Universidade Federal do Tocantins - TO - Brasil \\ Vinícius Souza Ribeiro \\ Instituto Federal de Educação, Ciência e Tecnologia do Tocantins - TO - Brasil \\ Robert Bailis \\ School of Forestry \& Environmental Studies (Yale University) - Connecticut - EUA
}

\begin{abstract}
Resumo: A produção de biocombustíveis tem sido fortemente discutida no Brasil, levando o País a desenvolver políticas e implantar, no ano de 2004, o Programa Nacional de Produção e Uso de Biodiesel (PNPB), a fim de aumentar a participação de energias renováveis, bem como fomentar o desenvolvimento regional sustentável. Nesse contexto, o presente estudo busca avaliar a relação entre a condição de vida dos agricultores e a adoção do cultivo de oleaginosas no norte do Brasil, em uma região de transição entre o Cerrado e a Floresta Amazônica. Uma gama de indicadores socioeconômicos foi coletada entre os agricultores que cultivam soja na região. A teoria dos conjuntos fuzzy baseada na condição de vida dos agricultores e um modelo não linear probit foram utilizados para avaliar a inclusão de famílias rurais pobres na cadeia do biodiesel. Os resultados preliminares mostram uma relação negativa entre o nível de privação das famílias e a adoção do cultivo de soja.
\end{abstract}

Palavras-chave: Biocombustíveis; Inclusão Social; Desenvolvimento Regional Sustentável

\begin{abstract}
Biofuel production has been greatly discussed in Brazil. In 2004, some debates lead the country to develop new policies and implement the National Biodiesel Use and Production Program (PNPB), with the intent to increase the share of renewable energy and foster sustainable regional development. In this context, the present study aims to assess the linkages between family farmer's living standard and the adoption of oil seed activity in northern Brazil, in a region of transition between the Cerrado (Brazilian savanna) and the Amazon rain forest. Ranges of socio-economic indicators were collected among smallholders who cultivate soybean. A fuzzy logic set theory based on living standard criteria and a non-linear probit model was applied to assess the inclusion of poor rural families in the biodiesel chain. Preliminary results point towards a negative relation between the family degree of deprivation and adoption of oil seed activity, for the soybean production.
\end{abstract}

Keywords: Biofuels; Social Inclusion; Sustainable Regional Development

\footnotetext{
${ }^{1} \mathrm{O}$ artigo é parte do Projeto "Agroenergia e Desenvolvimento Sustentável no Tocantins: uma Abordagem a partir de Sistemas Agrícolas e Rurais" (CNPq 401598/2011-0), liderado pelo primeiro autor.
} 


\section{Introdução}

A preocupação quanto aos problemas ambientais como a degradação e a exaustão dos recursos naturais, além da poluição atmosférica e do aquecimento global, tem levado governos e cientistas a identificar alternativas e buscar soluções para mitigar tais problemas. Hayes \& Nadkarni (2001) e Alier (2002), por exemplo, apontam que problemas ambientais ocorrem tanto nos países desenvolvidos como em países em desenvolvimento, tanto em áreas urbanas como em espaços rurais, e podem ser considerados como uma consequência dos atuais padrões de consumo e produção. É nesse contexto que desde o início do século 21 um debate internacional vem ganhando cada vez mais expressão e está presente na maior parte das conferências mundiais sobre desenvolvimento sustentável, a saber: biocombustíveis, seus prós e contras (DUBOIS, 2008; FAO, 2008a; FAO, 2008c).

Em relação aos prós, os biocombustíveis podem auxiliar na mudança da matriz energética de um país, através do uso de uma fonte mais limpa e renovável de energia. Com base nisso, os biocombustíveis também podem gerar externalidades positivas, como a manutenção da prestação de serviços ambientais tais como o sequestro de carbono e a redução das emissões de carbono, por exemplo (FAO, 2008d; FAO, 2008e). Do ponto de vista socioeconômico, os biocombustíveis podem alavancar o desenvolvimento regional, bem como fomentar estratégias que reduzam e/ou aliviem a condição de pobreza, as chamadas estratégias "pró-pobres" (FAO, 2008b; FAO, 2008d).

Contudo, apesar das inúmeras vantagens, algumas questões surgiram em relação às possíveis externalidades negativas geradas pela produção de biocombustíveis. Essas incluem, por exemplo, a diminuição da produção e da oferta de alimentos, bem como os impactos negativos sobre os serviços ambientais e as mudanças climáticas (FAO, 2008a). Quanto à questão da oferta de alimentos, a principal preocupação é que os biocombustíveis possam competir com as culturas alimentares. Essa competição por terra se torna um problema especialmente quando algumas das culturas que atualmente são usadas para a alimentação humana sejam redirecionadas para a produção de biocombustíveis (FAO, 2008b). Com a conversão de terras agrícolas para a produção de biocombustíveis, impactos negativos significativos sobre a segurança alimentar poderão ser observados, no que hoje é chamado de dilema "alimento versus combustível" ("food vs. fuel") (FAO, 2008a; FAO, 2008e; COTULA et al., 2008).

Com base nessa discussão, e seguindo, portanto, o debate internacional sobre biocombustíveis, em 2004 o governo brasileiro lançou o Programa Nacional de Produção e Uso de Biodiesel (PNPB), construído sob um cenário de preços elevados do petróleo, de uma crescente demanda mundial por combustíveis de fontes renováveis, e pelas vantagens comparativas do país em recursos naturais. $O$ PNPB, considerado um programa interministerial, tem como alguns de seus objetivos: implantar um programa sustentável, promovendo a chamada inclusão social dos agricultores familiares; garantir preços competitivos, qualidade e oferta de sementes oleaginosas, sem que isso interfira na produção de alimentos; produzir biodiesel a partir de óleos vegetais de diferentes espécies, bem como incrementar a renda agrícola dos agricultores familiares (PNPB, 2005). 
No entanto, após aproximadamente uma década desde a sua implantação, os impactos e a eficácia do programa permanecem pouco claros, sobretudo quanto à inclusão dos agricultores familiares mais carentes na cadeia do biodiesel. Nesse sentido, o presente estudo busca analisar e compreender as relações entre a produção de sementes oleaginosas e inclusão social, seguindo a concepção da Economia Verde Inclusiva, tema amplamente discutido durante a Conferência Rio +20 , que ocorreu no mês de junho de 2012 na cidade do Rio de Janeiro, Brasil. A Economia Verde Inclusiva concentra-se principalmente na intersecção entre o social e a economia, sendo um dos pilares na busca efetiva do Desenvolvimento Regional Sustentável.

Baseado nessa discussão, o artigo, portanto, tem como objetivo principal avaliar se o PNPB está sendo eficaz quanto ao cumprimento de sua meta da inclusão social. A inclusão social é aqui entendida como um processo que visa oferecer oportunidades de acesso às pessoas mais excluídas dentro de um sistema e, portanto, que beneficiam todos, e não apenas aqueles que possuem as melhores condições de acesso a bens e serviços (SILVER \& MILLER, 2002; PIERSON, 2002).

Espera-se que os resultados e a discussão apresentados aqui possam subsidiar os governos regional e federal, a fim de assegurar que o programa nacional de biodiesel possa, de fato, cumprir seus objetivos, sobretudo, de inclusão social daqueles que atuam na agricultura familiar na região da Amazônia Legal Brasileira. Além disso, os resultados apresentados podem e devem desempenhar um papel importante na integração de esforços para consolidar uma nova alternativa econômica para os agricultores familiares, buscando, como isso, o Desenvolvimento Regional Sustentável.

\section{Os biocombustíveis no Brasil}

\subsection{0 biodiesel}

Conforme a Lei no 11.097/2005, o biodiesel é um biocombustível derivado de biomassa renovável para uso em motores a combustão interna com ignição por compressão ou, conforme regulamento, para geração de outro tipo de energia, que possa substituir parcial ou totalmente combustível de origem fóssil (BRASIL, 2005). Ou seja, o biodiesel nada mais é que um biocombustível para ser utilizado em carros ou caminhões, in natura ou misturado, produzido a partir de biomassa, alternativo ao uso do diesel mineral e que, por ser biodegradável, causa um menor impacto ambiental (RIBEIRO, 2012).

O biodiesel é percebido como uma alternativa à utilização do diesel fóssil, uma vez que mantém as propriedades técnicas de funcionamento desse último sem que haja significativos impactos negativos, diretos e indiretos, sobre o meio ambiente (VIANNA, 2006). Holanda (2003), por sua vez, alerta que pode haver necessidade de adaptação em motores de ciclo de diesel; contudo, estes não são significantes ou onerosos e destaca que, devido às características físico-químicas semelhantes ao óleo diesel mineral, o biodiesel pode ser utilizado puro ou 
misturado em quaisquer proporções. Ainda segundo Holanda (2003), dada a dimensão continental do território brasileiro e suas condições edafoclimáticas, o país possui uma vantajosa condição de exploração em larga escala da biomassa para fins energéticos. Nessa linha o Brasil é reconhecidamente um dos países que detém as condições mais favoráveis de absorver boa parte da demanda global por energia limpa no século XXI.

\subsection{O Programa Nacional de Produção e Uso de Biodiesel (PNPB)}

No ano de 2003, diversos setores do governo brasileiro, a partir de um esforço interministerial, reuniram-se para discutir a viabilidade do uso do biodiesel como alternativa energética em âmbito nacional. Adicionalmente, foi recomendado o fomento sustentável que abrange temas como o desenvolvimento regional e a inclusão social, no sentido de serem norteadores para as futuras ações quanto à produção e ao uso do biocombustível. Outra questão levantada no relatório diz respeito ao não privilégio de processos produtivos, matérias-primas e escalas de produção em nível industrial (FINCO \& DOPPLER, 2011).

A partir das discussões e recomendações realizadas por diferentes setores do governo, em dezembro de 2004 o governo federal lançou oficialmente o Programa Nacional de Produção e Uso de Biodiesel (PNPB). O programa visa implantar, de forma sustentável, a produção e o uso de biodiesel, com enfoque, sobretudo, na inclusão social e no desenvolvimento regional (GARCEZ, 2008). Outros objetivos específicos do PNPB são a garantia de preços competitivos, suprimento e qualidade para o biodiesel, além da produção diversificada a partir de diferentes oleaginosas. Esses dois objetivos revelam a preocupação da sustentabilidade por parte do programa, na medida em que, ao buscar preços competitivos e oferta constante, cria condições no mercado para o biodiesel prosperar sem necessidade de recorrentes subsídios governamentais.

Em janeiro de 2005, o governo federal lançou o marco legal do PNPB, a Lei no 11.097/2005 (BRASIL, 2005), que introduziu compulsoriamente o biodiesel na matriz energética brasileira e fixou a obrigatoriedade da mistura de biodiesel ao diesel mineral (hoje em $5 \%$, também chamado de B5), com prazo de cumprimento no horizonte de oito anos. Ao estabelecer um prazo intermediário de três anos para o cumprimento de parte da meta, o governo criou um mercado inicial com potencial de comercialização de milhões de litros ao ano do biocombustível (ANP, 2013).

\subsection{O Selo Combustível Social (SCS)}

Simultaneamente ao lançamento do PNBP, o governo federal, através do Decreto $n^{\circ}$ 5.297, de 06/12/2004, instituiu o Selo Combustível Social (SCS) como instrumento de incentivo à inclusão dos agricultores familiares na cadeia produtiva do biodiesel. Um dos critérios para a concessão do selo às empresas produtoras de 
biodiesel, por parte do Ministério de Desenvolvimento Agrário (MDA), é a compra de parte da matéria-prima proveniente dos estabelecimentos enquadrados no Programa de Nacional de Fortalecimento da Agricultura Familiar (PRONAF) com fins de produção do biocombustível.

As unidades industriais produtoras de biodiesel, certificadas com o selo, devem, segundo a Portaria $n^{\circ}$ 60, de 06 de setembro de 2012 (MDA, 2012), comprar um mínimo, em percentagem, de matéria-prima da agricultura familiar, num percentual que varia de acordo com a região no Brasil. Para fins de uso, manutenção e concessão do SCS, devem ser celebrados previamente contratos com os agricultores familiares ou com suas cooperativas agropecuárias, seguindo condições e critérios mínimos estabelecidos na Instrução Normativa $\mathrm{n}^{\circ} 1$ de 19/02/2009 (MDA, 2009). Esses contratos devem garantir aos agricultores familiares desde assistência técnica, de responsabilidade das empresas produtoras do biodiesel, até garantia de formação do preço, ex-ante, da matéria-prima a ser entregue (com horizonte de variação positiva do preço vis-à-vis critérios préestabelecidos no contrato).

Essas estratégias lançadas pelo SCS inegavelmente têm um caráter inovador, sobretudo se comparadas à relativa ausência de metas e estratégias de integração da agricultura familiar ao circuito produtivo de biocombustíveis, como foi o caso do Proálcool (ainda que não tenha sido a essência do Proálcool) ${ }^{2}$. Porém, são necessários estudos mais aprofundados a fim de analisar até que ponto o PNPB está de fato promovendo a inclusão social da agricultura familiar na cadeia produtiva. Nesse sentido, as pesquisas apresentadas por Garcez (2008) e Finco (2010) apontam e sinalizam justamente para a ineficácia do programa quanto à inclusão socioprodutiva das famílias mais carentes na cadeia do biodiesel.

\section{Aspectos metodológicos e área de estudo}

A metodologia dos sistemas agrícolas e rurais, adotada por Finco (2010), foi utilizada neste estudo. Tal metodologia descreve o sistema agrícola familiar como sendo composto principalmente por três setores: (1) agrícola, per se; (2) não agrícola; e (3) doméstico. Esses setores, segundo o autor, estão ligados através de relações internas, levando em consideração as necessidades, objetivos, problemas e expectativas dos agricultores familiares sobre o futuro. Somado a isso, tal metodologia busca avaliar o sistema agrícola e rural de forma holística e sistêmica, através dos chamados indicadores de condição/padrão de vida (/iving standard indicators). Esses indicadores permitem uma profunda compreensão do funcionamento de um sistema agrícola e rural, uma vez que abordam aspectos econômicos, sociais, ambientais, demográficos, culturais e de saúde, dentre outros.

As fontes de informação utilizadas para realizar a pesquisa e obter os

\footnotetext{
${ }^{2}$ Mais detalhes podem ser vistos em Finco (2010).
} 
resultados incluem dados primários e inquéritos com agricultores familiares ${ }^{3}$. É na região de transição do Cerrado para a Floresta Amazônica, dentro do Estado do Tocantins, que a pesquisa foi realizada. O Tocantins está localizado na região norte do Brasil, e faz parte da chamada Amazônia Legal. Está situado em uma zona de transição, apresentando clima e vegetação da Floresta Amazônica $(15 \%$ do território) e Cerrado (85\% do território) (IBAMA, 2008). Essa área de transição, chamada zona ecotonal, é o lar de comunidades tradicionais (agricultores familiares, indígenas, e quilombolas) e inclui rica biodiversidade, a qual é responsável por inúmeros serviços ambientais. Por esta razão, estudos científicos e pesquisas na área são extremamente importantes.

Com base nessa questão, e a fim de obter as informações adequadas e relevantes para a pesquisa, uma macrorregião de estudo foi selecionada na parte centro-sul do estado do Tocantins, como se pode verificar na Figura 1.

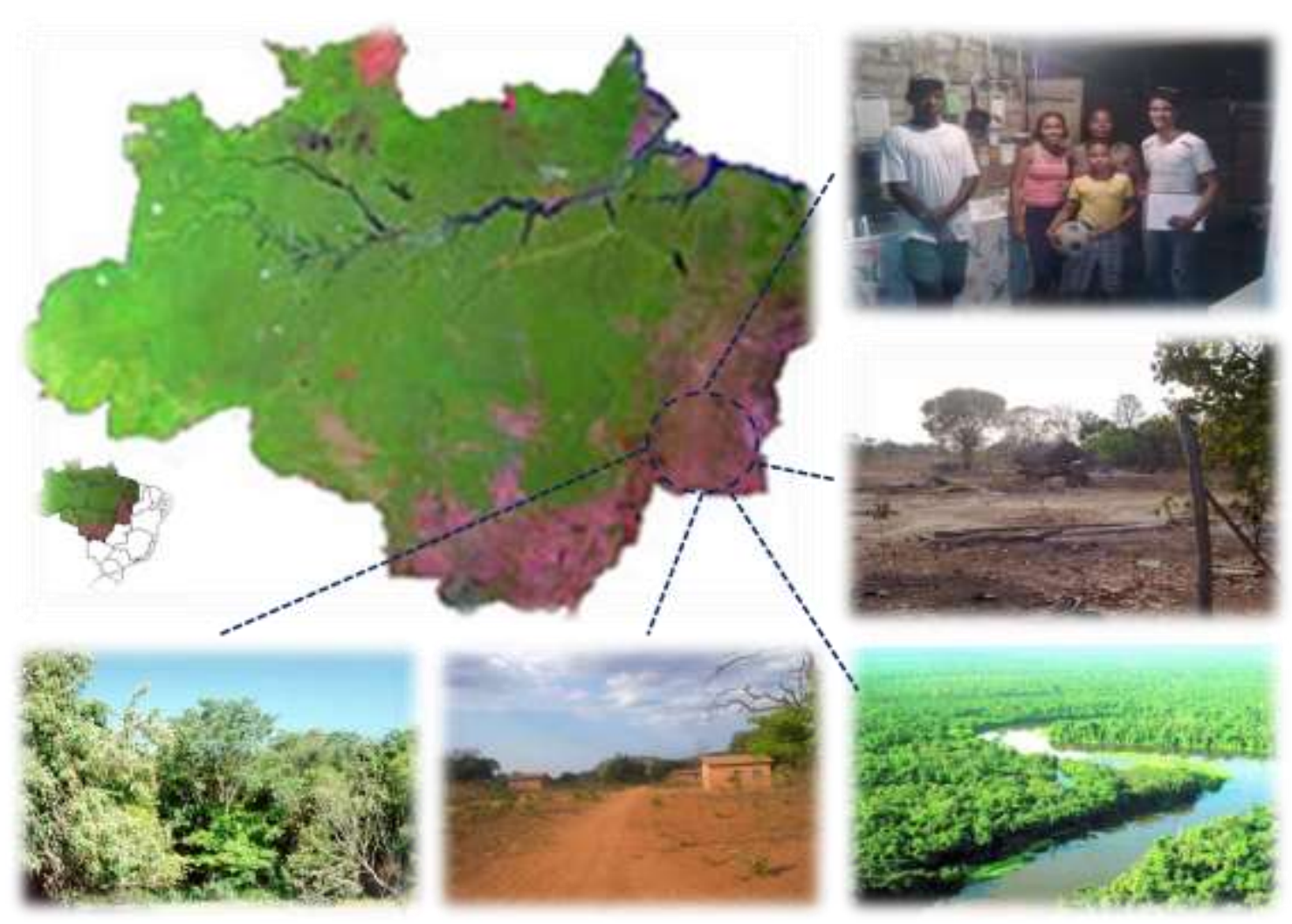

Figura 1. Macrorregião onde a pesquisa foi realizada no Estado do Tocantins Fonte: arquivo dos autores.

Já a maior parte dos dados secundários foi coletada na literatura sobre o tema. Os dados primários foram coletados no período compreendido entre os anos de 2012 e 2013, a partir de uma amostra de 62 famílias de agricultores familiares.

\footnotetext{
${ }^{3}$ Entende-se, no artigo, agricultura familiar como a agricultura realizada por proprietários rurais, onde o núcleo familiar é essencialmente a mão de obra utilizada nas atividades econômicas agrícolas, e cuja propriedade possua menos de 4 (quatro) módulos fiscais.
} 
Os dados coletados foram baseados em entrevistas com os agricultores sobre atividades agrícolas da safra 2011/2012, não agrícolas e domésticas, realizadas através de um questionário estruturado. O inquérito familiar foi realizado com agricultores familiares, que cultivavam sementes oleaginosas, e com agricultores familiares que não as cultivavam ${ }^{4}$. Isso se fez necessário, uma vez que um dos objetivos da pesquisa é compreender a relação entre adoção do cultivo de sementes oleaginosas e pobreza rural.

A teoria dos conjuntos fuzzy (ou lógica fuzzy) e análises de regressão não linear probit também foram utilizadas no estudo. Como mencionado anteriormente, o objetivo da presente pesquisa foi avaliar se houve, efetivamente, a inclusão social das famílias de agricultores mais carentes na cadeia produtiva do biodiesel, um dos pilares do PNPB. Para tal, foi construído um índice multidimensional de pobreza, que além da renda familiar, levou em consideração outros indicadores de privação das famílias. Esse índice foi utilizado como proxy para determinar quais famílias eram consideradas as mais carentes na região em questão. A partir do conhecimento desse atributo, foi estimado um modelo probit com o objetivo de inferir se as famílias mais carentes estavam, efetivamente, participando da cadeia produtiva do biodiesel e, portanto, sendo socialmente incluídas na atividade, no Estado do Tocantins.

\subsection{Teoria dos conjuntos fuzzy}

De acordo com Doppler (2004), sistemas agrícolas e rurais são sistemas complexos, e que são caracterizados pela interdependência e pela interação entre diferentes agentes. Estas interações normalmente resultam em efeitos não lineares, que são difíceis de avaliar, sobretudo, devido aos comportamentos que aparecem quando pequenas informações são reagrupadas em sistemas maiores, que as compõem. Assim, os sistemas agrícolas e rurais devem ser estudados e analisados de forma holística e sistêmica.

Portanto, um dos procedimentos mais promissores na investigação de sistemas agrícolas e rurais parece repousar na utilização de métodos que busquem captar e agrupar diferentes medidas de incerteza e de informações. É nesse contexto que se enquadra a teoria dos conjuntos fuzzy (REYS, 2003). O objetivo desta teoria é fazer valer o grau de adesão de um caso específico a um conjunto, e não apenas determinar a sua posição em um continuum em relação a outros casos. Nos estudos de sistema agrícolas e rurais, por exemplo, ao invés de mensurar a renda familiar de forma absoluta, pode-se enfatizar um grau de pertinência ao conjunto de agricultores familiares que são financeiramente seguros. Neste contexto, o conceito de conjunto fuzzy está relacionado a uma classe com um continuum de graus de adesão.

\footnotetext{
${ }^{4}$ A listagem com os agricultores produtores de soja foi encaminhada pela usina de biodiesel. Para fins de determinação do grupo de não produtores de sementes oleaginosas, o procedimento adotado foi de entrevistar também agricultores familiares que apresentassem características semelhantes aos produtores de sementes oleaginosas (neste caso, a soja) e, preferencialmente, vizinhos de propriedade.
} 
Com base nisso, a determinação das variáveis de entrada, através de termos linguísticos é referida como fuzzificação (ZADEH, 1972). Para isto, o grau real de adesão de variáveis de entrada é determinado para cada termo lingüístico da variável correspondente. Dados dos agricultores entrevistados, por exemplo, são apresentados e o processo de inferência é iniciado, o qual consiste em três subfunções: agregação, ativação, e acumulação. Finalmente, após o processo de inferência, o qual fornece uma função de pertinência, como consequência pode-se converter esse resultado em um número absoluto através da defuzzificação (ZADEH, 1965). O número absoluto gerado pelo processo de defuzzificação, por sua vez, deverá proporcionar uma boa representação das informações contidas no conjunto fuzzy.

A teoria dos conjuntos fuzzy foi escolhida porque permite a construção de um índice multidimensional de pobreza que (ao contrário de uma medida tradicional de pobreza) não só leva em consideração a situação material dos agricultores familiares, mas também capta as suas condições gerais de vida (MICELI, 1998) permitindo, com isso, que a relação entre inclusão social e produção de oleaginosas seja analisada e discutida com o devido aprofundamento. Muitas vezes, na abordagem tradicional, a pobreza é medida por uma linha de pobreza, ou seja, todos os indivíduos cuja renda não ultrapasse certo nível são declarados pobres (COSTA, 2002, DEUTSCH \& SILBER, 2005; BETTI e VERMA, 1999). Apesar de a abordagem tradicional apresentar seus méritos, como o de estabelecer os níveis de pobreza relativa e absoluta, ela não consegue capturar uma série de características (grau de pertencimento, nível de privação, dentre outros) que podem ser relevantes na compreensão de um fenômeno multidimensional como a pobreza.

Neste contexto, a teoria dos conjuntos fuzzy aplicada a estudos de pobreza pode ser considerada uma abordagem adequada por permitir a construção de um índice que leva em consideração o grau de adesão do individuo, que pode variar entre 0 (sem adesão, ou não pobre) e 1 (total adesão ou completamente pobre). Além disso, fuzzy é uma análise ampla, incluindo uma série de indicadores de qualidade de vida, ao mesmo tempo em que adota ferramentas matemáticas que permitem captar nuâncias de um fenômeno multidimensional (CHELI \& LEMMI, 1995).

Se uma determinada variável é medida em uma escala ordinal, por exemplo, a quantidade inerente aos seus níveis crescentes de privação deve ser levada em consideração. De acordo com Cerioli \& Zani (1995), isso pode ser facilmente feito através da atribuição de valores $\Psi_{j}^{(1)}\left(r=1,2, \ldots s_{j}\right)$ para as categorias $S_{j}$ de $X_{j}$, tal que:

$$
\Psi_{j}^{(1)}<\cdots<\Psi_{j}^{(r)}<\cdots<\Psi_{j}^{\left(s_{j}\right)}
$$

Se $\Psi_{j}^{\min }$ e $\Psi_{j}^{\max }$ são os valores correspondentes aos limites de um indivíduo 
ser considerado não pobre e pobre, respectivamente, ou seja, quando $\Psi_{j}^{\text {min }}$ corresponde às melhores condições de vida e $\Psi_{j}^{\max }$ corresponde às piores condições de vida em uma determinada região rural, por exemplo, as associações ou graus de pertinência podem ser expressos como:

$$
u_{\Xi_{j}}(i)=\left\{\begin{array}{l}
0 \\
\frac{\Psi_{i j}-\Psi_{j}^{\min }}{\Psi_{j}^{\max }-\Psi_{j}^{\min }} \\
1
\end{array}\right.
$$

$$
\begin{array}{r}
\text { se } \Psi_{i j}=\Psi_{j}^{\min } \\
\text { se } \Psi_{j}^{\min }<\Psi_{i j}<\Psi_{j}^{\max } \\
\text { se } \Psi_{i j}=\Psi_{j}^{\max }
\end{array}
$$

Onde $\Psi_{i j}$ é o valor recebido por um indivíduo i a partir do indicador $\xi_{\mathrm{ij}}$. Com essa especificação, a adesão (pertinência) aumenta linearmente com 0 agravamento da condição de pobreza. Alternativamente, a especificação trapezoidal é geralmente utilizada para variáveis contínuas (de natureza quantitativa), e postula que a função de pertinência para o conjunto de indivíduos pobres pode ser definida através da fixação de um valor $y^{\prime}$ até o ponto em que um indivíduo é definitivamente pobre. Em adição, um valor y", em que acima dele, um indivíduo é definitivamente considerado como não pobre, por exemplo:

$$
u_{\Xi_{j}}(i)=\left\{\begin{array}{lr}
1 & \text { se } 0 \leq y \leq y^{\prime} \\
\frac{y^{\prime \prime}-y}{y^{\prime \prime}-y^{\prime}} & \text { se } y^{\prime}<y \leq y^{\prime \prime} \\
0 & \text { se } y>y^{\prime \prime}
\end{array}\right.
$$

Nesse caso, abaixo de $\mathrm{y}^{\prime}$, um indivíduo é considerado totalmente pobre e acima de y", um indivíduo é considerado completamente não pobre. Para valores entre $y^{\prime}$ e $y^{\prime \prime}$, a função de pertinência assume valores em $[0,1]$, e parece natural torná-la uma função decrescente do indicador de privação.

Com base nisso, um índice fuzzy de pobreza (IFP) é construído através da média das funções de pertinência individual tais que IFP $\in[0,1]$, o qual representa a proporção de participação de indivíduos no subconjunto fuzzy de pobres.

$$
I F P=\frac{1}{n} \sum_{i=1}^{n} u_{p}(i)
$$

De acordo com Cerioli e Zani (1995), a abordagem fuzzy mencionada aqui tem o mérito de fornecer um retrato mais realista da pobreza do que a abordagem tradicional (dicotômica), que é baseada na renda dos indivíduos. Além disso, a 
teoria dos conjuntos fuzzy permite capturar as diversas facetas de um padrão de vida individual, além de salientar os diferentes graus em que cada indivíduo pode ser considerado pobre (BANTILAN et al., 1992). Nesse sentido, a metodologia dos conjuntos fuzzy é utilizada a fim de auxiliar a análise da relação entre pobreza rural e inclusão social de agricultores familiares na cadeia produtiva do biodiesel.

\subsection{Modelo probit}

Neste estudo, a relação entre pobreza rural e a adoção do cultivo de sementes oleaginosas é estimada utilizando-se o índice fuzzy de pobreza (IFP) descrito anteriormente. Com o objetivo de identificar e verificar o sentido da relação entre o IFP e a adoção de tal atividade, foram estimados dois modelos não lineares de regressão (probit), tendo variáveis binárias como dependentes, expressando a adoção ou não do cultivo de sementes oleaginosas, e o IFP como variável independente, expressando as situações de pobreza rural.

Nas variáveis dependentes, em todos os casos, toma-se como valor igual a zero quando os agricultores familiares não adotam o cultivo de sementes oleaginosas, e valor igual a um quando os agricultores familiares adotam tal atividade. Por outro lado, a variável independente utilizada (IFP) permite a caracterização das condições socioeconômicas dos agricultores familiares. Como regra geral, valores mais elevados desta variável apontam para piores condições de vida dos agricultores (maior grau de pobreza rural ou privação). Assim, quando o sinal do coeficiente estimado nos modelos apresentados abaixo for positivo, a relação entre pobreza rural e inclusão social é direta, ou seja, maior pobreza relaciona-se a maiores chances de adoção do cultivo de sementes oleaginosas. Por outro lado, quando o coeficiente é negativo, a relação é inversa, ou seja, maior o grau de pobreza relaciona-se com menores chances de adotar o cultivo de sementes oleaginosas e, portanto, de haver inclusão social desses agricultores familiares na cadeia produtiva do biodiesel.

$\mathrm{Na}$ presença de variáveis dependentes binárias, o modelo mais simples é o modelo linear de probabilidade, estimado pelo método de mínimos quadrados ordinários. Neste caso:

$$
\beta_{0}+\beta_{1} x_{1}+\ldots+\beta_{k} x_{k}
$$

Sendo $p$ a probabilidade de $y$ assumir o valor igual a um:

$$
E(y)=p=\beta_{0}+\beta_{1} x_{1}+\ldots+\beta_{k} x_{k}
$$

\footnotetext{
${ }^{5}$ Baseado em Greene (2008) e Hill et al., (2008).
} 
No modelo linear de probabilidade, os coeficientes estimados expressam o efeito de variações unitárias nas variáveis independentes sobre a probabilidade da variável dependente assumir o valor um. O problema é que estes efeitos são constantes, e à medida que $x_{\mathrm{i}}$ aumenta, a probabilidade $p$ continua a aumentar (quando $\beta_{\mathrm{i}}$ é positivo, caso contrário continua a diminuir) a uma razão constante. Entretanto, como $0 \leq p \leq 1$, é impossível ter uma taxa constante de crescimento.

Além disto, o modelo linear de probabilidade apresenta erros heterocedásticos, tal que os coeficientes estimados não são eficientes. Assim, os testes de hipóteses e intervalos de confiança podem ser inválidos.

Para contornar estes problemas, consideram-se os modelos não lineares probit e logit. Nesses casos, a inclinação não é constante. As probabilidades são restringidas ao intervalo $[0,1]$, pela utilização de funções densidade de probabilidade. A função probit está relacionada com a distribuição de probabilidade normal padronizada, enquanto a função logit está relacionada com a distribuição logística.

Como são modelos não lineares, a estimação dos coeficientes deve ser feita pelo método de máxima verossimilhança. Em geral, os coeficientes estimados nos modelos probit e logit são ligeiramente diferentes e a escolha entre eles pode ser feita de acordo com a conveniência. No presente artigo, o modelo probit foi o escolhido, considerando então que os erros têm distribuição normal.

Assim,

$$
p=F\left(\beta_{0}+\beta_{1} x_{1}+\cdots+\beta_{k} x_{k}\right)
$$

onde novamente $p$ é a probabilidade de $y$ assumir valor igual a um e $F$ é a função probit, não linear nos $\beta_{\mathrm{i}}$.

O efeito de uma variação unitária em $x_{\mathrm{i}}$ sobre a probabilidade $p$ de $y$ ser igual a um é dado por:

$$
\frac{\partial p}{\partial x_{i}}=\frac{\partial F_{(t)}}{\partial_{(t)}} \cdot \frac{\partial t}{\partial x_{i}}=f(.) \beta_{i}
$$

A partir disso,

(a) como $f(\cdot)$ é uma função densidade de probabilidade, seu valor é sempre positivo. Logo, o sinal de $\partial p / \partial x_{\mathrm{i}}$ é determinado pelo sinal de $\beta_{\mathrm{i}}$;

(b) à medida que $x_{\mathrm{i}}$ varia, o valor de $f($.) também varia. Dessa forma, o efeito de uma variação unitária nas variáveis independentes sobre a probabilidade da variável dependente depende dos níveis das variáveis independentes. Portanto, para estimar o efeito dos coeficientes do modelo probit é necessário escolher algum nível para as variáveis independentes como referência. 


\subsection{Indicadores de condição de vida: multidimensionalidade}

Para fins de cálculo do índice de pobreza multidimensional, utilizou-se uma série de indicadores de condição de vida (living standard indicators). Em adição, a teoria dos conjuntos fuzzy foi aplicada para a construção de um índice de pobreza baseado nestes indicadores. Contudo, antes de discutir os resultados encontrados para este objetivo proposto, serão apresentados os indicadores utilizados para a construção do índice de pobreza através da metodologia dos conjuntos fuzzy.

\subsubsection{Renda per capita e estoque de máquinas}

O indicador de renda per capita anual foi escolhido para apontar o nível de renda gozado por cada família, considerando a soma das rendas agrícolas e não agrícolas. Essas rendas devem ser trazidas para o período ex-ante ao cultivo da soja. Com base nisto, sobretudo para o grupo de famílias produtoras da oleaginosa, foi necessário estimar a renda agrícola anterior às áreas plantadas nas propriedades. Conforme a teoria dos conjuntos fuzzy, existe a necessidade de se estabelecer limites inferiores e superiores de renda per capita para que as famílias sejam consideradas mais ou menos (ou totalmente) pobres ou não pobres. Sendo assim, o limite superior (mais distante da pobreza) utilizado foi de $R \$ 20.503,00$ e o limite inferior (mais próximo da pobreza) foi de $R \$ 1.624,00$, por ano. Os dois limites foram, respectivamente, determinados a partir da média das vinte maiores rendas e vinte menores rendas. Quanto ao estoque de máquinas (também ex ante no caso dos agricultores produtores de soja), com valor superior a um salário mínimo vigente em 2012 ( $R \$ 622,00)$, foi atribuído o valor 0 para as famílias que possuíam estoque de maquinários superior a esse valor, e 1 no caso contrário.

\subsubsection{Anos de estudo e capital social}

O indicador anos de estudo apresentou valores entre 1 a 9 , sendo o valor 1 atribuído à família cujo chefe tinha nível superior incompleto e 9 para analfabeto. Os limites superiores e inferiores foram, portanto, 1 e 9, respectivamente. Quanto ao capital social, o mesmo foi medido pela participação ou não em associações, sindicatos, igrejas, etc. Para as famílias que participavam em mais de uma instituição, foi atribuído o valor 0; 0,5 para as famílias que participavam de apenas uma instituição; e 1 para as que não participavam de nenhuma instituição.

\subsubsection{Infraestrutura da casa}

Para quantificar a qualidade de vida habitacional das famílias, o indicador de construção da casa atribuiu o valor 0 para as famílias que tinham moradias de alvenaria, e 1 para as que não tinham. Outro indicador utilizado, que também 
revela a saúde sanitária da família, foi a existência (ou não) de banheiro dentro da residência. A água encanada na moradia também foi utilizada como indicador, sendo atribuídos os valores 0 (casa com água encanada) e 1 (casa sem água encanada).

\subsubsection{Fator de aglomeração}

O fator de aglomeração apresentou valores que variaram de 0,5 (limite inferior) a 5 (limite superior), em que tais valores representam a relação de quartos na casa por habitantes residentes.

\subsubsection{Bens duráveis}

A posse de bens duráveis variou de 1 (limite inferior) a 6 (limite superior), sendo 6 atribuído à família que possuía todos os oito bens analisados (televisão, celular, telefone fixo, rádio, computador, geladeira, fogão, máquina de lavar ou tanquinho).

\subsubsection{Meios de transporte}

Quanto aos meios de transporte utilizados pelas famílias, foram atribuídos valores que variaram de 1 a 4 , sendo considerado o valor 1 para as famílias que utilizavam, exclusivamente, transporte coletivo (em geral ônibus), 2 (famílias que tinham bicicleta), 3 (famílias que tinham moto) e 4 (famílias que tinham carro).

\subsubsection{Segurança alimentar}

Quanto à segurança alimentar familiar, foram realizados três questionamentos: (1) Já percebeu falta de alimentos em casa e precisou realizar compras?; (2) Aconteceu de ter que se arranjar com apenas alguns alimentos para comer porque não tinha recursos para aquisição no mercado?; (3) Aconteceu de alguém da família deixar, efetivamente, de se alimentar (café da manhã, almoço ou janta) porque não havia comida suficiente em casa? Para todos os questionamentos foram atribuídos valores 1 (resposta "sim") e 0 (resposta "não"). Dessa forma, para cada indicador a família era considerada pobre se a resposta fosse sim, e não pobre, caso respondesse negativamente ao questionamento. 


\subsection{8 Água potável}

Foram realizadas perguntas às famílias quanto à avaliação da qualidade da água para consumo, estabelecendo-se valores: 1 (ruim ou péssima), 2 (não tão boa), 3 (razoável), 4 (boa) e 5 (muito boa ou ótima), considerando-se 5 para o limite superior e 1 para o limite inferior. Quanto à origem da água, os valores variaram de 1 (limite inferior) a 3 (limite superior), sendo considerado 3 para a água de origem de companhia de abastecimento/saneamento, 2 para a oriunda de poços artesianos, e 1 para outras fontes (minas, córregos, riacho, lagos, etc.).

\subsubsection{Saúde familiar}

Para os indicadores de saúde familiar, foram consideradas a autoavaliação da condição de saúde dos membros da família, a existência de problemas crônicos de saúde, bem como a avaliação do atendimento médico utilizado pela família. Para o questionamento quanto à condição de saúde familiar, foram utilizados valores que variaram de 1 (limite inferior) a 5 (limite superior), que para uma condição ruim ou péssima de saúde (valor 1); não tão boa (valor 2); razoável (valor 3); boa (valor 4); e muito boa ou ótima (valor 5). Quanto à existência de problemas crônicos de saúde, foram estabelecidos valores 0 (não têm), 0,5 (apenas um problema) e 1 (mais de um problema). Nesse indicador, a família considerada pobre foi aquela que apresentou valor igual a 1, e vice-versa. Em relação à avaliação do atendimento de saúde (em geral pública), foram estabelecidos limites de 1 a 5, seguindo a classificação de 1 (ruim ou péssima), 2 (não tão boa), 3 (razoável), 4 (boa) e 5 (muito boa ou ótima).

\subsubsection{Grau de dependência}

Quanto ao grau de dependência (relação entre membros com menos de 14 anos e mais de 60 anos, divididos pelo número de membros entre 14 e 60 anos, ou seja, membros familiares ativos) foram estabelecidos os limites de 0 a 3 , sendo considerada pobre a família com grau de dependência 3, e vice-versa.

Com isso, após o estabelecimento dos indicadores de padrão de vida, o índice de pobreza foi estimado através da teoria dos conjuntos fuzzy. Por sua vez, após a estimação do índice fuzzy de pobreza (IFP), os resultados referentes às relações entre o IFP e a adoção do cultivo de sementes oleaginosas por parte dos agricultores familiares foram estimados através de modelos de regressão não lineares probit. É importante salientar que a metodologia dos conjuntos fuzzy foi aplicada a cada indicador de padrão de vida (aqui chamados de indicadores de privação), com o objetivo de estimar o grau de adesão dos agricultores familiares a estes indicadores. 
Tabela 1. Natureza dos indicadores de privação $\left(\xi_{j}\right)$

\begin{tabular}{lll}
\hline \multirow{2}{*}{ Indicadores de privação $\left(\xi_{\mathrm{j}}\right)$} & \multicolumn{2}{c}{ Definição } \\
\cline { 2 - 3 } & Natureza do indicador & Modelo fuzzy \\
\hline Renda per capita & Contínuo & Trapezoidal \\
Estoque de máquinas & Dicotômico & Tradicional \\
Anos de estudo & Politômico & Linear \\
Capital social & Politômico & Linear \\
Casa de alvenaria & Dicotômico & Tradicional \\
Banheiro na casa & Dicotômico & Tradicional \\
Água encanada & Dicotômico & Tradicional \\
Fator de aglomeração & Politômico & Linear \\
Bens duráveis & Politômico & Linear \\
Meios de transporte & Politômico & Linear \\
Falta de alimentos & Dicotômico & Tradicional \\
Falta de alguma refeição & Dicotômico & Tradicional \\
Poucos alimentos disponíveis & Dicotômico & Tradicional \\
Qualidade da água & Politômico & Linear \\
Origem da água & Politômico & Linear \\
Autoavaliação da saúde & Politômico & Linear \\
Problema crônico de saúde & Politômico & Linear \\
Avaliação do sistema de saúde & Politômico & Linear \\
Grau de dependência & Politômico & Linear
\end{tabular}

Fonte: elaboração própria.

\section{Resultados e discussão}

Após o cálculo de cada indicador, para cada família, pôde-se observar que o grupo de famílias não produtores de soja apresentaram, na média, valores superiores (mais próximos de 1) em comparação ao grupo de famílias produtores de soja, apontando que, na média, o primeiro grupo contempla o grupo de famílias mais carentes, se comparado ao segundo grupo, na região em questão. Portanto, a priori, pode-se auferir que o grupo de famílias não produtores de soja é relativamente mais carente que o grupo de famílias produtores da oleaginosa, sinalizando, ainda que, de forma inicial, a inclusão social é, no mínimo, duvidosa na região em questão (Figura 2). Cabe ressaltar que as estimativas referentes, sobretudo, a renda e a estoques de máquinas, foram realizadas levando-se em consideração o cenário ex-ante a produção de sementes oleaginosas (neste caso a soja), a fim de minimizar qualquer ressonância de impacto desta atividade nos indicadores de privação. 


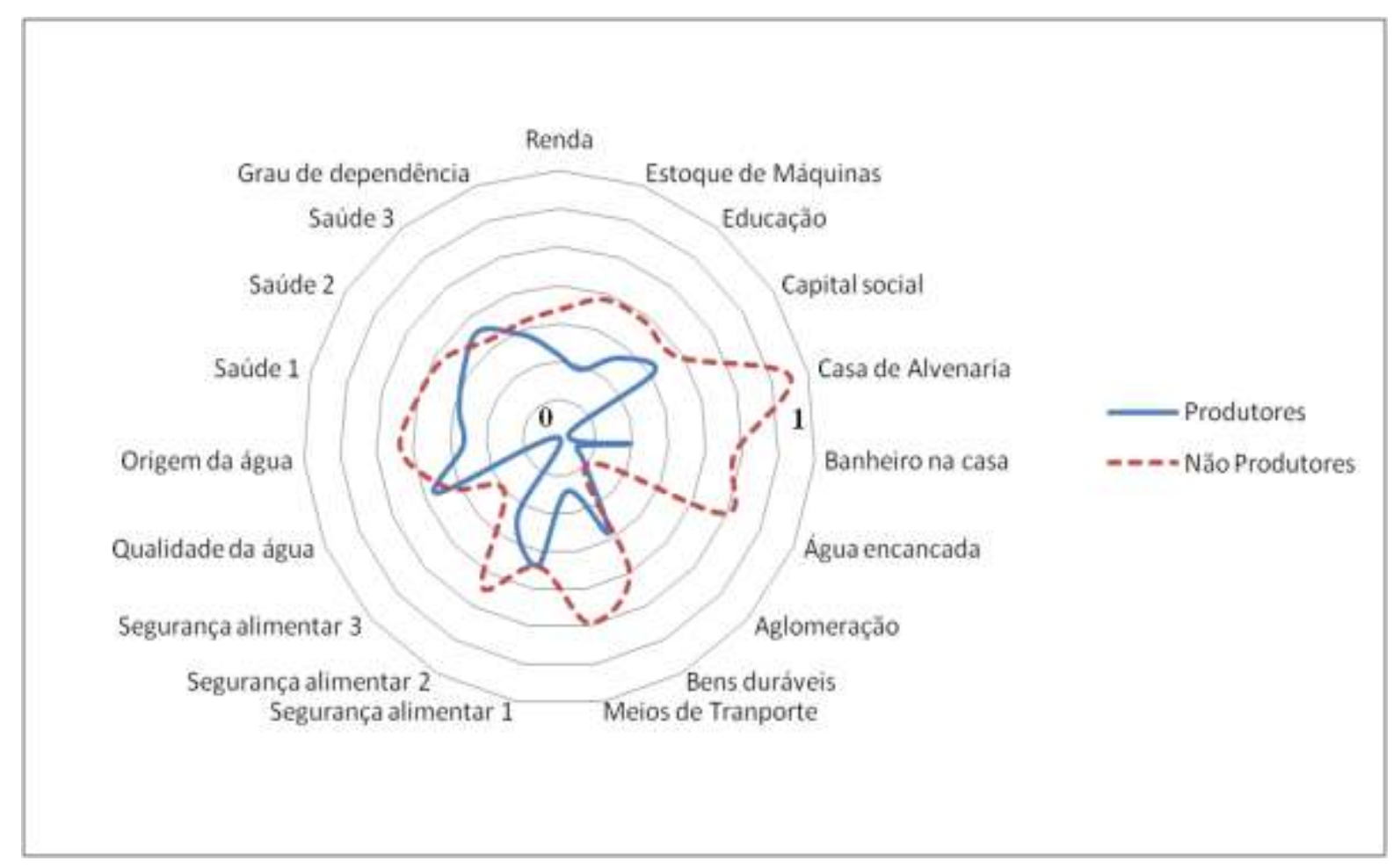

Figura 2. Indicadores de privação dos agricultores familiares na região de estudo Fonte: resultados da pesquisa.

Em seguida, um índice fuzzy de pobreza foi estimado para a região de estudo, ou seja, compreendendo todos os agricultores familiares (produtores e não produtores de soja). Isso é importante, a fim de que a robustez do índice fuzzy de pobreza seja verificada e validada. Vale reforçar que a teoria dos conjuntos fuzzy é aplicada através de diferentes modelos (trapezoidal, linear e tradicional), bem como através do estabelecimento de diferentes valores-âncora. Nesse sentido, é importante notar que quanto menor o número de agricultores privados com relação a determinado indicador de privação, maior é o peso deste indicador na composição do IFP. Isso acontece devido ao fato de que o sistema de pesos utilizados no presente estudo dá mais importância aos indicadores de privação associados com menores sintomas da pobreza, uma vez que os indivíduos têm, por natureza, um forte sentimento de privação quando não possuem acesso a um bem ou serviço bastante difundido entre seus pares (MICELI, 1998).

A interpretação do índice fuzzy é complexa, porque combina indicadores de diferentes naturezas. Ainda assim, ele pode gerar informações importantes sobre as condições de vida dos agricultores familiares. Contudo, como o objetivo do estudo é avaliar a inclusão social, ou seja, estimar a relação entre pobreza rural e adoção do cultivo de sementes oleaginosas. Após a estimativa do índice fuzzy de pobreza para a região, os agricultores familiares foram separados em "pobres" e "não pobres", de acordo com seu próprio índice fuzzy de pobreza (IFP). Com base nisso, os agricultores familiares que possuem, na média, um IFP acima de 0,29 foram considerados pobres. Somado a isso, com o objetivo de compreender os vínculos entre o índice fuzzy de pobreza dos agricultores familiares e a inclusão 
social destes na cadeia produtiva do biodiesel, um modelo de regressão não linear probit foi formulado, a fim de estimar a relação entre a pobreza rural e a adoção do cultivo de sementes oleaginosas.

Tabela 2. Índice fuzzy de pobreza estimado para a região de estudo

\begin{tabular}{lcc}
\hline \multirow{2}{*}{ Indicadores de privação $\left(\xi_{j}\right)$} & \multicolumn{2}{c}{ Definição } \\
\cline { 2 - 3 } & $\begin{array}{c}\text { Proporção fuzzy de pobres } \\
\overline{\boldsymbol{u}}\left(\boldsymbol{\xi}_{\boldsymbol{j}}\right)\end{array}$ & $\begin{array}{c}\text { Pesos } \\
\boldsymbol{\omega}_{\boldsymbol{j}}\end{array}$ \\
\hline Renda familiar per capita & 0.633 & 0.022 \\
Estoque de máquinas & 0.613 & 0.023 \\
Anos de estudo & 0.524 & 0.031 \\
Capital social & 0.411 & 0.043 \\
Casa de alvenaria & 0.419 & 0.042 \\
Banheiro na casa & 0.290 & 0.059 \\
Água encanada & 0.161 & 0.088 \\
Fator de aglomeração & 0.890 & 0.006 \\
Bens duráveis & 0.445 & 0.039 \\
Meios de transporte & 0.226 & 0.071 \\
Falta de alimentos & 0.468 & 0.036 \\
Falta de algumas refeições & 0.290 & 0.059 \\
Poucos alimentos disponíveis & 0.032 & 0.165 \\
Qualidade da água & 0.294 & 0.059 \\
Origem da água & 0.403 & 0.044 \\
Autoavaliação de saúde & 0.286 & 0.060 \\
Problema crônico de saúde & 0.444 & 0.039 \\
Avaliação do sistema de saúde & 0.484 & 0.035 \\
Grau de dependência & 0.189 & 0.080 \\
IFP (Índice fuzzy de pobreza) & 0.290 & \\
\hline Fonte: resulos da pesqusa & &
\end{tabular}

Fonte: resultados da pesquisa.

Como o objetivo da presente pesquisa é avaliar a inclusão dos agricultores familiares pobres na cadeia produtiva do biodiesel, isto é, estimar a relação entre pobreza rural e o cultivo de soja para produção de biocombustível, a especificação do modelo probit é dada por:

$$
Y_{j}=\alpha+\beta X_{j}+\mu_{j} \quad(\mathrm{j}=1, \ldots, \mathrm{n})
$$

Onde a variável dependente $Y$ refere-se à adoção de sementes oleaginosas $(Y=1)$ ou não adoção $(Y=0)$, $\alpha$ e $\beta$ são os parâmetros da equação, $\mu$ é o termo estocástico e a variável independente $\mathrm{X}$ é uma variável dummy, onde: 1 refere-se a quando uma família de agricultores familiares é considerada pobre; e 0 quando uma família não é considerada pobre (com base no índice fuzzy de pobreza, IFP, mencionado anteriormente) e, portanto: 


$$
X=\left\{\begin{array}{c}
1 \text { se } X_{j} \geq 0.290 \\
0 \text { se caso contrário }
\end{array}\right.
$$

A variável explicativa, expressada pelo índice fuzzy de pobreza, permite caracterizar a condição de vida dos agricultores familiares na região em questão. Valores mais baixos do IFP indicam uma condição de vida melhor. Assim, quando o sinal do coeficiente estimado a partir do modelo probit é positivo, a relação entre pobreza rural e inclusão social é direta, ou seja, a probabilidade de os agricultores familiares pobres adotarem o cultivo de sementes oleaginosas é maior do que os seus homólogos não pobres na mesma região. Portanto, a meta da inclusão social e a estratégia de desenvolvimento "pró-pobre" estão sendo eficazes.

Porém, quando o coeficiente for negativo, a relação é inversa, ou seja, os agricultores familiares pobres têm, em média, menor probabilidade de adotar o cultivo de sementes oleaginosas do que os agricultores familiares não pobres na mesma região e, portanto, a eficácia do PNPB no que tange à meta da inclusão social é, no mínimo, ambígua e duvidosa.

Os resultados do modelo probit podem ser vistos na Tabela 3.

Tabela 3. Resultados do modelo de regressão probit

\begin{tabular}{lccc}
\hline & \multicolumn{3}{c}{ Região de estudo (soja) } \\
\cline { 2 - 4 } & Coef. & EP & Estat. Z \\
\hline Constante & 0.6985 & 0.2387 & $-93^{*}$ \\
IFP & -1.6432 & 0.3639 & $-4.52^{*}$ \\
N. obs & & 62 \\
LR $\chi^{2}$ & & $22.7^{*}$ \\
Pseudo R & & 0,26 \\
\hline
\end{tabular}

Fonte: resultados da pesquisa.

Notas: EP = erro padrão. ${ }^{*}$ Significativo a $1 \%$.

Pode-se observar que os coeficientes, na região do estudo, são significativos a $1 \%$. A probabilidade Qui-quadrado $\left(\chi^{2}\right)$ também é significativa a $1 \%$. Percebese que o sinal do coeficiente IFP é negativo, ou seja, os agricultores familiares pobres têm, em média, uma menor probabilidade de adotar o cultivo de sementes oleaginosas, quando comparados a seus pares que não são considerados pobres nesta mesma região. Aqui, é evidente que o desenvolvimento "pró-pobre" não está ocorrendo, uma vez que os agricultores mais carentes não estão participando da cadeia produtiva do biodiesel e, portanto, a inclusão social defendida pelo PNPB não está sendo percebida. 
Quando estimado o efeito marginal do coeficiente IFP, observa-se que ser um agricultor familiar pobre nesta região, por exemplo, diminui a probabilidade de adoção do cultivo de sementes oleaginosas (e, portanto, de não ser incluído na cadeia produtiva do biodiesel) em 58\%, em média, quando comparado a um agricultor familiar não pobre, o que representa um obstáculo para a promoção do chamado Desenvolvimento Regional Sustentável.

Os resultados da pesquisa indicam, portanto, que o PNPB não está sendo efetivo, enquanto política de inclusão social, ou seja, as famílias de agricultores mais carentes não estão participando, efetivamente, da cadeia produtiva do biodiesel. Sob outra ótica, o PNPB está atuando como uma política de exclusão social à medida que reduz significativamente a probabilidade de uma família carente fazer parte da cadeia produtiva do biocombustível. Em parte, o que pode estar ocorrendo é uma escolha, ex-ante, por parte das empresas produtoras de biodiesel quanto às famílias (ou o perfil de famílias) que farão parte do programa, isto é, possivelmente as empresas estão optando por escolher famílias que tenham um nível de escolaridade maior, estoque mínimo de capital, localização privilegiada, ou até um histórico com o plantio da oleaginosa, o que reduziria gastos com assistência técnica e a probabilidade de perda da produção.

Nessa perspectiva, haveria um descompasso entre a meta governamental de incluir as famílias mais carentes na cadeia produtiva e o interesse das empresas em manter o selo, reduzir gastos e ter garantia de entrega da soja para produção do biodiesel, o que provavelmente está atrelado às parcerias com famílias que tenham um conhecimento prévio do cultivo da oleaginosa e estoque de capital mais bem estruturado. Em síntese, as empresas não estariam interessadas em contratar agricultores familiares mais carentes, mas sim os agricultores mais consolidados e que minimizassem seus custos de transação e, consequentemente, de produção do biocombustível. A própria forma como as parcerias surgem reforçam esta possibilidade de escolha prévia das famílias, uma vez que, majoritariamente, é a empresa quem vai até as famílias de agricultores familiares, e não o contrário.

Há, portanto, a necessidade de uma reformulação da base de incentivos à inclusão social da agricultura familiar na cadeia nacional de biodiesel, que atualmente se restringe ao selo social. Além da adoção de novos mecanismos que estimulem a inclusão social, existe a necessidade de se promover um maior estímulo ao desenvolvimento de pesquisas, não só por parte do setor público, que viabilize economicamente o uso de outras matérias-primas que não somente a soja, hoje responsável por cerca de $80 \%$ de toda a matéria-prima utilizada para a produção nacional de biodiesel. Isso possibilitaria a participação de um número maior de famílias no PNPB, o que facilitaria a inclusão das famílias mais carentes, levando-se em consideração que o cultivo da soja não é tradicional na agricultura familiar brasileira em geral, pelo menos nas regiões mais carentes e menos desenvolvidas do país. 


\section{Considerações finais}

Apesar do fato de o PNPB possuir objetivos louváveis de promover a inclusão social e o desenvolvimento regional, os resultados apresentados demonstram uma ineficácia com relação à meta da inclusão social. Os resultados sugerem a ausência de mecanismos de políticas específicas, o que resultou no não cumprimento, de forma efetiva, de um dos objetivos principais do PNPB. Com base nisso, é imperativo dizer que o envolvimento de diferentes stakeholders, centros de pesquisa, empresas e governos locais parece ser uma condição sine qua non para superar as deficiências nos mecanismos estabelecidos pelo PNPB, com o objetivo de minimizar as lacunas da política nacional de biodiesel e, portanto, alcançar a inclusão social e o desenvolvimento regional sustentável.

Cabe ressaltar que este estudo é inédito na região e os resultados são extremamente importantes para ajudar a obter um método apropriado para os governos regionais e nacionais subsidiarem a produção de energia limpa, sem agredir o meio ambiente local ou a produção de alimentos e, portanto, ajudando o Brasil a alcançar o desenvolvimento regional sustentável. A produção de sementes de óleo em pequena escala pode agora ser mais bem avaliada em outras partes da Amazônia Legal Brasileira, uma vez que o estudo destaca um dos temas mais discutidos no debate sobre a bioenergia: produção de sementes oleaginosas e sua precedência sobre a inclusão socioprodutiva dos agricultores familiares.

Como contribuições para futuras pesquisas a partir das limitações deste trabalho, sugere-se analisar os impactos ambientais da produção do biodiesel através de uma análise do ciclo de vida do produto, assim como a realização de estudos com dados primários que busquem analisar a inclusão dos agricultores familiares mais pobres na cadeia do biodiesel através do uso de outras oleaginosas e em diferentes regiões do país. Certamente, a complementação dessas informações, de forma científica, são essenciais para a construção de um arcabouço importante para a avaliação e (re)formulação das políticas públicas ligadas não somente à agroenergia no Brasil, mas à própria promoção da Economia Verde Inclusiva e, consequentemente, do Desenvolvimento Regional Sustentável.

\section{REFERÊNCIAS}

ALIER, J. M. 2002. The Environmentalism of the Poor. University of Witswatersrand. Disponível em http://www.wrm.org.uy/actors/WSSD/alier.pdf (acessado em 26 de Outubro de 2009).

Agência Nacional de Petróleo, Gás Natural e Biocombustíves (ANP). Dados estatísticos. Disponível em: http://www.anp.gov.br. Acesso em: 05 de ago. de 2013.

BANTILAN, M. C. S.; BANTILAN Jr, F. T.; CASTRO, M. M. 1992. Fuzzy sub set theory in the Measurement of Poverty. In: Journal of Philippine Development, $\mathrm{n}$. 34 , vol. XIX. 
BETTI, G.; VERMA, V. K. 1999. Measuring the degree of poverty in a dynamic and comparative context: a multi-dimensional approach using fuzzy set theory.

Proceedings of the ICCS-VI, Lahore, Pakistan, August 27-31, Vol. 11, pp. 289-301.

BRASIL. Lei No 11.097 de 13 de Janeiro de 2005. Disponível em:

<http://nxt.anp.gov.br/NXT/gateway.dll/leg/leis/2005/lei\%2011.097\% 20\% 202 005.xml?f $=$ templates $\$ f n=$ default.htm \&sync $=1 \& v i d=$ anp: $10.1048 /$ enu $>$. Acesso em: 08 de ago. de 2013.

CERIOLI, A.; ZANI, S. 1995. A Fuzzy Approach to the Measurement of Poverty. In: Dagum, C. and Zenga, M. (eds.). Income and Wealth Distribution, Inequality and Poverty, Springer Verlag, Berlin, p.272-284

CHELI, B.; LEMMI, A. 1995. A "Totally" Fuzzy and Relative Approach to the Multidimensional Analysis of Poverty. In: Economic Notes, vol. 24, pp. 115-134.

COSTA, M. 2002. A Multidimensional Approach of the Measurement of Poverty. IRISS Working Paper Series n. 2002-05. Available at http://iriss.ceps.lu/documents/irisswp28.pdf (verified 26 October 2009).

COTULA, L.; DYER, N.; VERMEULEN, S. 2008. Fuelling exclusion? The biofuels bomm and poor people's access to land. Disponível em http://www.iied.org/pubs/pdfs/12551IIED.pdf (acessado em 26 de Outubro de 2009).

DEUTSCH, J.; SILBER, J. 2005. Measuring Multidimensional Poverty: An Empirical Comparison of Various Approaches. In: Review of Income and Wealth. Series 51, n. 51.

DOPPLER, W. 2004. Farming and Rural Systems Approaches. Published Lecture Material. Hohenheim University, Stuttgart, Germany.

DUBOIS, O. 2008. How Good Enough Biofuel Governance Can Help Rural Livelihoods: Making sure that Biofuel Development Works for Small Farmers and Communities. FAO, Rome, Italy.

FAO (Food and Agriculture Organization of the United Nations). 2008a.

Bioenergy, food security and Sustainability - Towards an International Framework. Disponível em http://www.fao.org/fileadmin/user_upload/foodclimate/HLCdocs/HLC08-inf-3E.pdf (acessado em 26 de Outubro de 2009).

FAO (Food and Agriculture Organization of the United Nations). 2008b. Climate Change, Bioenergy and Food Security: Civil Society and Private Sector Perspectives. Disponível em http://www.fao.org/fileadmin/user_upload/foodclimate/HLCdocs/HLC08-inf-6E.pdf (acessado em 26 de Outubro de 2009). 
FAO (Food and Agriculture Organization of the United Nations). 2008c. Climate Change, Bioenergy and Food Security: Options for Decision Makers identified by Expert Meetings. Disponível em http://www.fao.org/fileadmin/user_upload/foodclimate/HLCdocs/HLC08-inf-5E.pdf (acessado em 26 de Outubro de 2009).

FAO (Food and Agriculture Organization of the United Nations). 2008d. Bioenergy Policy, Markets and Trade and Food Security. Disponível em $\mathrm{ftp}: / / \mathrm{ftp} . f a o . o r g / d o c r e p / f a o / m e e t i n g / 013 / a i 788 e . p d f$ (acessado em 26 de Outubro de 2009).

FAO (Food and Agriculture Organization of the United Nations). 2008e. The State of Food and Agriculture. Biofuels: prospects, risks and opportunities. Disponível em http://www.fao.org/docrep/011/i0100e/i0100e00.htm (acessado em 26 de Outubro de 2009).

FINCO, M.V.A. Bioenergy Economics: An Analysis of Oil Seed Farming and Biodiesel Production in the Brazilian Savannah. Tese (Doutorado em Economia) Instituto de Economia Agrícola e Ciências Sociais nos Trópicos e Sub Trópicos, Universidade de Hohenheim, 2010.

FINCO, M.V.A.; DOPPLER, W. The Brazilian biodiesel program and regional development: cases from northern Brazil. REDES, Santa Cruz do Sul, v16, n.13, p.215-241.

GARCEZ, C. A. G. Uma Análise da Política Pública do Programa Nacional de Produção e Uso de Biodiesel (PNPB). Brasília, DF, 2008. 171p. Dissertação (Mestrado em Desenvolvimento Sustentável) - Centro de Desenvolvimento Sustentável, Universidade de Brasília, 2008.

GREENE, W. H. 2008. Econometric analysis. Sixth edition.

HAYES, A.; NADKARNI, M. V. 2001. Poverty, Environment and Development. Studies of four countries in the Asia Pacific Region. Disponível em http://unesdoc.unesco.org/images/0012/001219/121999e.pdf (acessado em 26 de Outubro de 2009).

HILL, R. C.; GRIFFITHS, W. E.; LIM, G. C. 2008. Principles of Econometrics. Third edition.

HOLANDA, A. O biodiesel e a inclusão social. 2003. Disponível em:

<http://www.sfiesc.org.br/artigos/tecnologia/BIODIESEL_2003.pdf> Acesso em: 25 ago. 2013.

LELLI, S. 2001. Factor Analysis vs Fuzzy Sets Theory: Assessing the Influence of Different Techniques on Sen's Functioning Approach. Discussion Paper. Faculty of Economics and Applied Economics, Katholieke Universiteit Leuven. Disponível em 
http://www.econ.kuleuven.ac.be/ew/academic/econover/Papers/DPS0121.pdf (acessado em 12 de outubro de 2009).

Ministério do Desenvolvimento Agrário (MDA). Portaria ministerial $n^{\circ} 60$ de 06 de setembro de 2012. 06/09/2012. Instrução Normativa $n^{\circ} 1$ de 19 de fevereiro de 2009. 19/12/2009.

MICELI, D. 1998. Measuring poverty using fuzzy sets. Discussion paper no. 38. National Centre for Social and Economic Modeling. University of Camberra.

PNPB. 2005. Programa Nacional de Produção e Uso de Biodiesel. www.biodiesel.gov.br/programa.html. (verified 12 November 2009).

PIERSON, J. 2002. Tackling Social Exclusion. (Social Work Skills Series).

REYS, M. A. 2003. Farming and Rural Systems Analyses in Forest Margins: An Application of Fuzzy Theory. The case of West Tocantins, Brazil. In: DOPPLER, W. and BAUER, S. (Eds.) Farming and Rural Systems Economics. Vol. 48.

Weikersheim, Margraf Verlag.

RIBEIRO, V.S. Política Pública do Biodiesel e o Desenvolvimento Local Sustentável para a Agricultura Familiar. XII Colóquio Internacional sobre o Poder Local: Desenvolvimento e Gestão Social de Territórios, 2012. Anais do XII Colóquio Internacional sobre o Poder Local: Desenvolvimento e Gestão Social de Territórios.

SILVER, H.; MILLER, M. 2002. Social Exclusion: The European Approach to Social Disadvantage. Issue of Poverty and Race. Disponível em http://www.ag.ohiostate.edu/ hcrd/people/staff/Social\% 20Exclusion\% 20The\% 20European\% 20app roach.pdf (acessado em 15 de março de 2010).

VIANNA, F.C. Análise de Ecoeficiência: Avaliação do Desempenho EconômicoAmbiental do Biodiesel e Petrodiesel. São Paulo, SP, 2006. 183p. Dissertação (Mestrado em Engenharia) - Escola Politécnica, Universidade de São Paulo, 2006. 


\section{Sobre os autores}

\section{Marcus Vinicius Alves Finco}

PhD em Economia Agrícola (Universitaet Hohenheim, Alemanha). Professor Adjunto da Universidade Federal do Tocantins (UFT, Brasil).

Email:marcus.finco@gmail.com

\section{Vinícius Souza Ribeiro}

Mestre em Desenvolvimento Regional (Universidade Federal do Tocantins, Brasil).

Endereço: AE 310 SUL (AESE 34) AV. LO-5, s/n - Setor Sul. CEP 77021-090 - Palmas - TO - Brasil

\section{Robert Bailis}

PhD em Recursos Energéticos (University of California/Berkeley, EUA). Professor Associado na School of Forestry \& Environmental Studies (Yale University, EUA).

E-mail: robert.bailis@yale.edu 